\title{
Evaluación de dos métodos para calificar la hidratación corporal en niños con diarrea aguda. Experiencia con enfermeras
}

\author{
Marion Feddersen W. ${ }^{l}$; Alicia Denegri C. ${ }^{2}$; Sandra Ponce F. ${ }^{2}$; \\ Flora Fernández G. ${ }^{2}$; Marcos Emilfork S. ${ }^{3}$

\section{Comparisson of two methods to asses hydration state by nurses in children with acute diarrhea}

\begin{abstract}
The need to ocurately assess hydration state in children with acute diarrhea by medical and paramedical personnel, arises from the fact that diartheal disease is stil an important cause of death in some places of the world. With the hypotesis that the method of Fortin and Parent used by nurses, improves the assessment of hydration of children with acute diarthea, 70 children 24 months of age or less were studied using this method and the usual clinical assessment. The gold standard was the weight gain ance the child was rehydrated. The prevalence of dehydration was $63,8 \%$. Mole and children of 6 months and more predominoled. The tested method misclassified $50 \%$ of the children, whereas usual clinical asessment did so in only $27.63 \%$ of them $(p<0,051$. Overestimation of dehydration was higher with the method of Fortin and Parent $\{47,4 \%$ vs $25 \%\}$ ip $<0,05$ ). Underestimation was equal for both methods. No iniluence of age or nutritional status in the evaltation of patients was observed. Usual dinical asessment showed higher specificity, positive predictive value and efficiency than Fortin Parent's method, and this lare, when used by nurses, does not improve the assessment of the first one.
\end{abstract}

(Koy words: body fluids, dehydralion, ocute diarrhea.)

La disponjbilidad de un método objetivo para valorar el estado de hidratación en niños con diarrea aguda ha sido motivo de preocupación de especialistas en el tema y de instituciones como la Organización Mundial de la Salud, ya que la adecuada calificación del estado de bidratación de un paciente con diarrea tiene una implicancia crucial en la terapéutica de la anormalidad fiiopatológica, y, por ende, en el pronóstico de la enfermedad diarreica ${ }^{1-9}$. La persistencia del cólera en muchos países, así como su aparición en áreas donde se creía erradicado, ha dado al tema una mayor relevancia.

La importancia de un método -supuestamente más objetivo- aumentaría si su rendimiento en el diagnóstico fuera mejor que el usado tradicionalmente ${ }^{47}$, cuando es aplicado por profesionales médicos y también paramédicos. Esto últi-

1. Departamento de Pediatna, Hospital Roberto del Rio.

2. Enfermera Universitaria. Clínica Sauta María.

3. Servicio de Pediatría, Clínica Santa María. mo, porque en algunos parses el manejo de los pacientes con diarrea aguda y deshidratación está en manos de personal paramédico ${ }^{8}+9$.

El método de Fortin y Parent ${ }^{4}$ procura clasificar los pacientes en tres categorías, según la siguientes calificaciones: 0 a 3 puntos, no deshidratados o con deshidratación leve; 4 a 8 puntos, deshidratados moderados; 9 a 17 puntos, deshidratados intensos (tabla 1). El método clínico tradicional valora los signos clínicos como tensión de la fontanela bregmática, sequedad de las mucosas, presencia o ausencia de ojos hundidos y lágrimas, así como el tiempo que tarda el pliegue realizado en la piel del enfermo en desaparecer. Todos estos signos confluyen en una integración que hace el evaluador para separar las categorías de pacientes no deshidratado, deshidratado leve, moderado e intenso $0^{4} .10,11$. Evaluado en médicos, el procedimiento tradicional ha resultado en proporciones de error entre $25,6 \%$ y $35,2 \%$. 12 . En 1992 no se pudo demostrar que el sistema de Fortin y Parent fue- 
Tabla 1

Método de Fortin y Parent para calificar el estado de hidratación

\begin{tabular}{|c|c|c|c|c|}
\hline & 2 & 1 & 0 & Puntaje \\
\hline Lengua & Seca & Ligeramente seca & Húmeda & $A=$ \\
\hline Pliegue & Persistente & Desaparece lento & Ausente & $\mathbf{B}=$ \\
\hline Globuto ocular & Muy hundidos & Hundidos leve & Normal & $\mathrm{C}=$ \\
\hline Fontanela & Muy hundida & Hundida leve & Plana & $\mathrm{D}=$ \\
\hline Extremidades & Frias y azules & Frias & Tibias & $\mathbf{E}=$ \\
\hline Est. neurológico & Apático o irritable & Lloroso. & Normal & $\bar{F}=$ \\
\hline Respiración & Profunda & Rápida & Tranquila & $\mathrm{G}=$ \\
\hline \multicolumn{4}{|c|}{ Si el niño estấ semicomatoso o muy irritable, con tlanto de tono agudo, agregue 3 al total } & $\mathbf{H}=$ \\
\hline Total & & & & \\
\hline
\end{tabular}

se superior al tradicional, cuando ambos eran empleados en niños con diarrea aguda por médicos entrenados. El porcentaje de error con dicho método fue de $41,4 \%$, ante $25,6 \%$ del tradicional $(p: 0,003)^{?}$. En la literaura chilena no se registra una experiencia que evalúe el método tradicional aplicado por enfermeras universitarias, como tampoco el de Fortin y Parent cuando es utilizado por personal paramédico.

Con la hipótesis que el método de Fortin y Parent, aplicado por enfermeras universitarias, mejora la calificación del estado de hidratación de niños con diarrea aguda, decidimos iniciar esta experiencia.

\section{Material y Método}

Suponiendo un error de $40 \%$ al aplicar el método tradicional por enfermeras, y con tá precisión de campo requerida para realizar este trabajo, se eligí el tamaño de la muestra considerando errores alfa $5 \%$ y beta $30 \%$. Para estos requisitos el tamaño de la muestra correspondía a 74 pacientes ${ }^{13}$. Ingresaron al estudio 76 niños hospitalizados en el servicio de pediacría de la Clínica Santa María, con el đíagnóstico de diarrea aguda. Todos eran menores de 24 Ineses. La gravedad de los pacientes incluyó desde casos sin deshidratación hasta otros intensamente deshidratados. El estándar ideal fue la ganancia de peso, una vez desaparecidos los signos de deshidratación y obtenida la estabiljzación det peso, lo que ocurriós siempre dentro de las primeras 24 horas desde el ingreso. La calificación del estado de hidratación inicial fue hecha por enfermeras universitarias del servicio, las cuales recibieron el entrenamiento suficiente para obtener la información requerida. Los pacientes provenían de consultas de sus médicos tratantes 0 del servicio de urgencia de la clínica. En general se trataba de pacientes de situación sociøeconórnica media o alta. La variación interobservador fue investigada en una sub- muestra de 15 enfermos tomados al azar, registrándose ésta en 9/15 enfermos, con una mediana de variación de 2 puntos y márgenes 0 a 5 puntos, con el método de Fortin $y$ Parent, mientras con el tradicional se encontro coincidencia en $12 / 15$ y diferencia no mayor a un grado en los tres casos restantes. Se definieron como bien hidratados los niños que no mostraban signos de deshidratación, cuyo peso varió menos de $2 \%$ en 24 horas. El estándar ideal se expresó como porcentaje del déficit, y contemplo los tramos de $5 \%$ o menos (deshidratación leve o ausente), 6 a $9 \%$ (deshidralación moderada) y $10 \%$ o más (deshidratacín intensa, con o sin choque hipovolémico). El peso fue medido con nif́o desnudo en balanza de palanca marca SECA (R), con sensibilidad de 10 gramos, empleándose la misma en cada pesada. El déficit se determinó según la fómula: deficicit (\%) = (peso hidratado - peso ingreso)/peso hidratado $x 100$. La estatura fue medida con niño en decúbito dorsal y antropometro. El estado de nutrición fue calificado de acuerdo a nomas vigentes ${ }^{14}$, según criterio peso/ tallh, con el paciente bien hidratado, empleando como estăndar el percentil 50 de las tablas del NCHS ${ }^{15}$

Eл el análisis de los resultados se comparó cada método con el estándar y luego ambos procedimientos entre sí, considerando la proporción de casos adecuada o inadecuadamente calificados; la influencia de la edad y el estado de la nutrición en la calificación; los niveles de sensibilidad, especificidad, valores predictivos y eficiencia ${ }^{16}{ }^{17}$. Con este objeto la información se ajustó a tablas de $2 \times 2$. En la comparacion de ambos metodos los pacientes hidratados se agruparon con los deshidratados leves, ante los niños que presentaban deshidratación moderada o intensa según el estándar ideal. Para el análisis de signos individuales en el metodo de Fortin y Parent. esto correspondió a los valores 0 ante 1 y 2 (tabla 1). Las pruebas estadísticas utiljzadas fueron chi cuadrado, diferencia de tasas, Fisher y Mc Nemar ${ }^{16-18}$.

\section{Resultados}

Setenta y seis niños ingresaron al estudio, predominando los de 60 más meses de edad $(92,1 \%) ; 53,9 \%$ eran varones, $53,9 \%$ tenian pe- 
so normal o sobrepeso, $40,8 \%$ desnutrición grado I y ninguno sufría desnutrición severa. En todos los casos la hidratación normal se alcanzó dentro de Jas 24 horas de haber ingresado. De acuerdo con el estándar ideal, $81,6 \%$ de los niños se consideraron bien hidratados o tenfan deshidración leve, sólo $1,3 \%$ tenían deshidratación intensa y la prevalencia global de deshidratación en la muestra fue $63,8 \%$.

La concordancia de los resultados obtenidos al comparar ambos métodos, con el estándar ideal, fue insatisfactoria, pues ellos subestimaron el grado de deshidratación en el 2,6\% de los casos, pero la sobreestimación fue de $47,4 \%$ para método de Fortin y Parent ante $25 \%$ con e1 tradicional ( $p<0,05$ ). Al comparar los dos métodos de clasificación con el señalado estándar ideal (tablas 2 y 3 ), puede observarse que e] método tradicional catalogó erróneamente a $27,63 \%$ de los pacientes, mientras el de Fortin y Parent lo hizo en $50 \%$ de los casos ( $p<0,05$ ).

La clasificación de los pacientes obtenida por los dos métodos en comparación resulto significativamente distinta $(\mathrm{p}<0,01)$, comprobándose que el tradicional clasifić bien a 18 pacientes mal ubicados con el de Fortin y Parent, mientras este último sólo mejor 6 la clasificación otorgada mediante el tradicional en sólo un caso (tabla 4). La edad y el estado de nutrición no afectaron la calificación del estado de hidratación comparando entre ambos méto$\operatorname{dos}(p>0,05)$.

La capacidad de ambos sistemas para discriminar entre pacientes normobidratados o con trastorno leve de pacientes con los que sufrian deshidratación moderada o intensa (tabla 5), fue alta y semejante en sensibilidad y valor predictivo negativo. Sin embargo, la especificidad, valor predictivo positivo y eficiencia fueron francamente menores con el de Fortin y Parent. De los rubros clínicos que este último procedimiento considera, destacaron con alta sensibilidad el estado neurológico, el aspecto del globo acular, el carácter del pliegue y la humedad de la lengua. La especificidad fue en general baja, siendo mayor para la fontanela y las extremidades. Llamó la atención el bajo valor predictivo positivo para la totalidad de los elementos clínicos que considera el método aludido, así como los altos valores predictivos negativos. La eficiencia varió entre $43,4 \%$ para el estado neurológico hasta $73,6 \%$ para el examen de la fontanela.
Tabla 2

Estado de bidratación en 76 riños hospilalizados. Comparación entre la clasificación según estándar ideal y método de Fortin y Parent

\begin{tabular}{lcccr}
\hline \multirow{2}{*}{$\begin{array}{l}\text { Fortin y Parent } \\
\text { (puntos) }\end{array}$} & \multicolumn{4}{c}{ Estándar ideal (\% déficit de peso) } \\
& $5 \mathbf{n}$ (n) & $\mathbf{6 - 9}(\mathrm{n})$ & $210(\mathrm{n})$ & Total \\
\hline $0-3$ & 30 & 1 & 0 & 31 \\
$4-8$ & 29 & 8 & 1 & 38 \\
$9-17$ & 3 & 4 & 0 & 7 \\
\hline Total & 62 & 13 & 1 & 76 \\
\hline
\end{tabular}

(chi cuadrado: 13,$746 ; p<0,01$ )

Tabla 3

Estado de hidratación en 76 niños hospitalizados. Comparación entre la calificación según el estándar ideal y el método clínico tradicional

\begin{tabular}{lrrrrr}
\hline Método tradicional & \multicolumn{3}{c}{$\begin{array}{c}\text { Estíndar deal } \\
\text { (\% déficit de peso) }\end{array}$} \\
& $\leq \mathbf{5}$ (n) & $\mathbf{6}-\mathbf{9}$ (n) & $\mathbf{\geq 1 0}$ (n) & Total \\
\hline Sin o leve deshidratación & 44 & 1 & 1 & 46 \\
$\begin{array}{l}\text { Moderada deshidratación } \\
\text { Intensa deshidratación }\end{array}$ & 18 & 11 & 0 & 29 \\
\hline Total & 0 & 1 & 0 & 1 \\
\hline
\end{tabular}

(chi cuadrado: 16,$27 ; \mathrm{p}<0,01$ )

Tabla 4

Clasificación del estado de hidratación en 76 nifros hospitalizados utilizando el método de Fortin y método clínico tradicional

\begin{tabular}{lccc}
\hline & \multicolumn{3}{c}{ Método de Fortin y Parent } \\
\hline $\begin{array}{l}\text { Método } \\
\text { tradicional }\end{array}$ & $\begin{array}{c}\text { Bien } \\
\text { clasificado }\end{array}$ & $\begin{array}{c}\text { Mal } \\
\text { clasificado }\end{array}$ & Total \\
\hline Bien clasificado & 37 & 18 & 55 \\
Mal clasificado & 1 & 20 & 21 \\
\hline Total & 38 & 38 & 76
\end{tabular}

(Según prueba de Mc Nemar, chi cuadrado: 1,$47 ; \mathrm{p}<0,01$ )

\section{Comentario}

La necesidad de valorar adecuadamente el estado de hídratación de pacientes con diarrea aguda, radica en que este trastorno sigue siendo 


\section{Tabla 5}

Sensibilidad. especificidad, valores predictivos, eficiencia y proporción de error de método tradicional (MCT) y método de Fortin y Parent (MFP) así como de sus componentes en 76 pacientes, como porcentajes según presencia e intensidad de la deshidratación

\begin{tabular}{lccccccccc}
\hline & MCT & MFP & Fontanela & Extrem. & Neurol. & Resp. & Gl. ocular & Pliegue & Lengaa \\
\hline Sensibilidad & 85,7 & 93 & 50,0 & 35,7 & 100,0 & 57,1 & 92,8 & 85.7 & 100,0 \\
Especificidad & 70,9 & 48 & 79,0 & 75,8 & 30,6 & 56,4 & 37,0 & 54,8 & 40,3 \\
VP + & 40,0 & 29 & 35,0 & 25,0 & 24,5 & 32,0 & 25,0 & 30,0 & 27,4 \\
VP - & 95,6 & 97 & 87,5 & 83,9 & 100,0 & 85,3 & 95,8 & 94,4 & 100,0 \\
Eficiencia & 73,6 & 57 & 73,6 & 68,4 & 43,4 & 56.5 & 47,3 & 60.5 & 51,3 \\
Proporción de error & 27,6 & 50 & & & & & & & \\
\hline
\end{tabular}

VP+ : valor predictivo posirivo

VP. : valor predictivo negativo

una causa importante de muerte en el mundo, principalmente por las pérdidas de agua y electrólitos que produce ${ }^{19}$. Conviene buscar un método que obvie lo subjetivo del examen tradicional y que pueda ser aplicado eficazmente tanto por médicos como por personal paramédico ${ }^{4,5,7.9,19}$.

Llama la atención en nuestra muestra el predominio de niños de edad igual o mayor a 6 meses, situación distinta a la observada durante muchos años en nuestros hospitales públi$\cos ^{7 \cdot 10 \cdot 12}$. Creemos que la explicación radica en que esta experiencia fue realizada en un hospital privado, con una población de enfermos distinta a la que suele concurrir a los hospitales públicos. La misma razón puede esgrimirse para explicar la ausencia de desnutridos intensos y el gran porcentaje de nin̄os eutróficos o con sobrepeso. La gran cantidad de pacientes bien hidratados o deshidratados leves y sólo un enfermo con deshidratación intensa, sin choque hipovolémico, es una distribución semejante a la encontrada en los hospitales públicos ${ }^{7,12,20}$, 10 que significa, en nuestra opinión, que la condición en que los niños son hospitalizados y el criterio de hospitalización son similares en ambos lugares. Esto no es de extrafiar, pues en el país hay consenso en que el paciente deshidratado debe ser tratado en un hospital, aunque el déficit sea leve ${ }^{10 .}$ 11, 14. La baja incidencia de deshidratación de grado mayor tampoco es un fenómeno propio de la muestra que se comenta, ya que en los últimos años el número de pacientes de nivel socioeconómico bajo que llega a los hospitales con deshidratación intensa y choque hipovolémico es inferior a $3 \% \mathrm{c}^{12}$.
Contrariamente a nuestra hipótesis, las enfermeras no calificaron con mayor precisión e] estado de la hidratación aplicando el método de Fortin y Parent, que con el tradicional, pues el porcentaje de error fue de casi el doble ( 50 vs $27,63 \% ; \mathrm{p}<0,05)$ y estuvo fuertemente influido por sobrevaloración del déficit, lo que en la práctica no resulta significativamente peligroso, por cuanto, si bien el paciente recibe un tratamiento más intenso que el necesario, el riesgo que esto implica es mínimo frente a los nocivos resultados de la subvaloración. El menor nivel de error del método tradicional guardó relación con la adecuada calificación que, al aplicarlo, se consigui 6 en aproximadamente la mitad de los casos mal catalogados mediante el de Fortin y Parent. Además, este último método sólo mejoró la calificación de menos de 5\% de los pacientes mal calificados por el tradicional.

La falta de influencia del estado nutricional y la edad sobre las calificaciones otorgadas por ambos métodos podría deberse al pequeño tamaño de la muestra, ya que el efecto de la desnutrición intensa sobre el método tradicional ha sido comprobado en estudios bechos en un hospital público, con una muestra más grande y mayor proporción de desnutridos ${ }^{10,12,20}$.

La baja especificidad, escaso valor predictivo positivo y poca eficiencia del método de Fortin y Parent son posiblemente consecuencias de la sobrevaloración del déficit que resulta de su empleo. Por otro lado el bajo valor predictivo positivo para la prevalencia de deshidratación en este estudio sugiere que la utilización del método en la comunidad, donde la prevalencia del trastorno es menor, disminuiría aún más su 
valor. El alto valor predictivo negativo para todos los rubros contemplados en el método de Fortin y Parent y los bajos valores predictivos positivos podrian deberse a la incidencia menor a $20 \%$ de deshidratación moderada e intensa. Estos resultados son semejantes a los de un trabajo anterior? ${ }^{7}$ en que ambos métodos fueron aplicados por médicos en pacientes de un hospital público, aun cuando las poblaciones estudiadas son diferentes.

En conclusión, el método tradicional calificó el estado de hidratación con menor error que el de Fortin y Parent. Además, con este último se sobrevaloró la intensidad de la deshidratación. sin mejorar la calificación del método tradicional, el que tuvo una sensibilidad prácticamente igual, pero mayor especificidad, valor predictivo positivo y eficiencia que el método de Fortin y Parent, por lo cual estos resultados no permiten comprobar nuestra hipótesis de trabajo.

\section{Resumen}

La necesidad de valorar adecuadamente el estado de hidratación en pacientes con diarrea aguda, por médicos y paramédicos, radica en que esta afección constituye aun una causa importante de muerte en el mundo. Con la hipoteis que el método de Fortin y Parent (método de Fortin y Parent), aplicado por enfermeras universitarias, mejoraría la calificación del estado de hidratación de niños con diarrea aguda, se estudiaron 76 niños de 24 meses o menos de edad, utilizando este método y el clínico tradicional, comparando ambos con un estándar ideal determinado por la ganancia de peso una vez hidratado el paciente. La prevalencia de deshidratación fue de $63,8 \%$. Hubo un predominio de varones $(53,9 \%)$ y de niños mayores o iguales a 6 meses $(92,1 \%)$. Con el método de Fortin y Parent se clasific 6 erróneamente a $50 \%$ de los niños, mientras con el tradicional esto sucedió en $27,63 \%$ ( $p<0,05$ ) de los casos. La sobreestimación de la deshidratación fue mayor con el método de Fortin y Parent $(47,4$ vs 25\%) ( $p<0,05$ ) y la subestimación fue la misma con ambos métodos $(2,6 \%)$. La edad y el estado nutricional no influyeron en la calificación del estado de hidratación. El método tradicional mostrố mayores especificidad, valor predictivo posi- tivo y eficiencia. En conclusion, cuando es utilizado por enfermeras, el método de Fortin y Parent no mejora la calificación obtenida con el método tradicional.

(Palabras clave: deshidratación, diarrea agu$\mathrm{da}$, líquidos corporales.)

\section{Agradecimientos}

A las enfermeras universitarias del servicio de Pediatría de la Clínica Santa María: G. Lucero C. y C. Alamos G., por su colaboración en este trabajo.

\section{Referencias}

1. Lanata C. Black R: El problema mundial de las diarreas. En: Torregrosa L. ed. Enfermedades diarreicas en el niña. 9' Ed. México: Ediciones Medicas del Hospital Infantil de México, 1988: 3-9.

2. Glass RI, Lew JF, Gangarosa RE, LeBaron CW, Shang $M$ : Estimates of morbidity and mortality rates for diarrheal diseases in American children. J Pediat 1991: 118: S27-\$33.

3. Organización Mundial de la Salud: Organización Panamericana de la Salud: Manual de tratamiento de la diarrea. Serie Paltex $N^{\circ} 13,1987$; I20-170.

4. Fortin J, Parent $M$ : Dehydration scoring system for infants. Trop Pediat Env Child Health 1978; 24; 110-114.

5. Hochman H, Grodin M. Crone R: Debydration, diabetic ketoacidosis and shock in the pediatric patient. Pediatr Clin North Am 1979; 30: 215-219.

6. Moto F: Deshidratación. En Torregrosa L. ed. Enfermedades diarreicas en el nifino. $9^{\mathbf{L}}$ Ed. México. Ediciones Médicas del Hospital Infantil de México 1988; 347.353.

7. Duffau G, Emilfork $M$ : Evaluación del estado de hidratación en nif́os hospitalizados por diarrea aguda. Comparación de dos métodos. Acta Pediatr Esp 1992; 50: $842-850$.

8. Organización Murdial de la Salud: Organizacion Panamericana de la Salud. Programa de Salud Materno Infantil. Control de las enfermedades diarreicas. Tratamiento de la diarrea $2^{*}$ ed. agosto 1987; 11-21.

9. World Health Organization: Programme for control of dianhael diseases, Seven programme report, 19881989. WHO/CDC $90 ; 34: 11-12$.

10. Duffak $G$ : Sindrome diarreico agudo en el lactante. Santiago de Chile: Ed. Andrés Bello, 1978; 137-156.

11. Duffau $G$. Emilfork $M$ : Metabolismo hidrosalino y balance ácido-base en el nifo. Santiago de Chile: Andrés Bello, 1989: 19-23.

12. Enilfork $M$. Duffau $G$, Godenberg $E$, Méndez $B$ : Caracterización del síndrome diarreico agudo del lactante. I, Aspectos Clínicos. Pediatría (Santiago) 1987; 30: 203-209.

13. Fleiss JL: Statistical Methods for Rates and Proportions. Second Ed. New York. John Wiley and Sons Ed. 
14. Aguiló C, Emparanza E. Rizzardini M: Normas de atención pediátrica. 3" ed. Santiago de Chile. Mediterraneo. 1989; 41-70

15. Nelson W: Textbook of Pediatrics. 12* ed., Philadelphia. WB Saunders Co: $1983 ; 27-35$.

16. Riegelman $R K$ : Studing a study and testing a test. Boston- Little Brown and Company, 1981; 99-100.

17. Dawson-Saunders B, Trapp RG: Procedjmientos para valorar diagnósticos. En: Bioestadística Médica. Ed Manual Modemo. México 1990: 265-282.
18. Haber A. Runyon R: Estadística General. México, Fondo Educativo Interamericano 1973; 185 . 240.

19. Organización Mundial de la Salud. Organización Panamericana de la Salud. Manejo del paciente con diarrea. 3* ed 1991; 1-25.

20. Emilfork $M$, Duffau $G$ : Validación de un método propuesto por la Organización Mundial de la Salud para calificar estado de hidratación en nitios con diarrea aguda. Acta Pediatr Esp (En prensa).

\section{AVISO A LOS AUTORES}

Por acuerdo del Comité Editorial, la Revista Chilena de Pediatría devol verá sin tramitar todos los trabajols que no den estricto cumplimiento al Reglamento de Publicaciones y a las Instrucciones de los Autores que se editan en cada número de la Revista. 